\title{
UNIFIED CONSTITUTIVE MODELING OF THREE ALLOYS UNDER A WIDE RANGE OF TEMPERATURE
}

\author{
Yukio Takahashi ${ }^{1}$ \\ ${ }^{1}$ Executive Research Scientist, Central Research Institute of Electric Power Industry, Japan
}

\begin{abstract}
Use of proper constitutive models is essential in evaluating structural integrity of various components using inelastic analysis. In particular, temperature-dependency of the deformation behaviour has a large influence on the result and needs to be taken into account in an appropriate manner. Three kinds of materials used in nuclear reactor vessels and their attachments were chosen to study their deformation behaviour under a wide range of temperature from room temperature to $1100^{\circ} \mathrm{C}$ and various strain rates, in order to model them in a unified way. Two kinds of temperature dependency corresponding to short range and long range obstacles to dislocation movement were introduced to the models, in order to describe the temperature and strain-rate dependent behavior in low-temperature and high-temperature regime connected by the intermediate regime where the temperature and strain-rate dependency was negligible. Systematic process was developed for optimizing the functions and constants included in the models. Reasonable agreements have been obtained between the model predictions and the experimental data for all the alloys studied.
\end{abstract}

\section{INTRODUCTION}

In the normal operating conditions, temperatures experienced by structural materials of light water reactor plants do not reach the level which can cause creep deformation. However, in Beyond Design Basis Events (BDBE) including severe accidents, temperatures may enter the range where temperature and strain rate effects on deformation and strength are significant. Constitutive models as well as failure criteria applicable to these conditions need to be developed in order to provide tools for evaluating structural integrity of components under such circumstances.

Here three representative alloys constituting pressure boundary of typical light water reactor plants are tested at a wide range of temperatures for obtaining monotonic tensile properties at various strain rates. Then constitutive models describing them are developed for utilization in the structural analyses for the components under BDBE. This paper outlines the constitutive models developed in comparison with test results.

\section{OUTLINE OF EXPERIMENT}

In this study, JIS SQV2A (equivalent to A533B)、SUS316 and Alloy 600 used in light water reactor plants were employed as test materials. They are hot-rolled plates with a thickness of $173 \mathrm{~mm}, 50 \mathrm{~mm}$ and $25 \mathrm{~mm}$, respectively. Standard round bar specimens with a test section of $6 \mathrm{~mm}$ diameter and $30 \mathrm{~mm}$ length were taken from the plates in the direction of final rolling.

Monotonic tensile tests were conducted $\mathrm{n}$ strain-control mode using an electrical-mechanical tensile testing machine with an electrical furnace. About 40 tests were performed on each material, changing temperature and strain rate parametrically. Testing temperature was varied between room temperature and $1100^{\circ} \mathrm{C}$ whereas the strain rate was changed in a range between $0.001 \% /$ and $1 \% / \mathrm{s}$. Values of inelastic strain deduced from total strain and stress values using Young's modulus were used for 
modelling. As the elastic strain rate is much smaller than inelastic strain rate except very early stage of the tests, total strain rate was regarded as the inelastic strain rate for the sake of simplicity.

\section{CONSTITUTIVE MODELING}

\section{Modeling of Stress at Start of Inelastic Deformation}

Initial deformation property represents the relationship between stress and inelastic strain rate when inelastic deformation starts. It is commonly considered that resistance against dislocation movement which is responsible for inelastic deformation under practical loading conditions consists of two elements; i.e. short-range obstacle coming from inter-atomic potential and long-range obstacle generated by interaction between dislocations as well as with precipitates in the matrix or grain boundaries. When maximum values of these components are denoted by $\sigma_{s r \text {,max }}$ and $\sigma_{l r \text {,max }}$, respectively, stress required for generating initial inelastic deformation conventionally called yield stress can be represented by the summation of these components as

$$
\sigma_{y}=\left(\frac{E}{E_{0}}\right) \sigma_{s r, \max }\left\langle 1-\left\{\frac{R T_{a b s}}{Q_{s r} E} \ln \left(\frac{\dot{\varepsilon}_{s 0}}{\dot{\varepsilon}_{\text {in }}}\right)\right\}^{1 / 1.5}\right\rangle^{2}+\left(\frac{E}{E_{0}}\right)\left(1+R_{D S A}\right) \sigma_{l r, \max }\left[1-\exp \left\{-\frac{\dot{\varepsilon}_{i n}}{\dot{\varepsilon}_{l 0}} \exp \left(\frac{Q_{l r}}{R T_{a b s}}\right)\right\}^{1 / n}\right]
$$

where $E$ is the Young's modulus calculated by

$$
E=g_{0}+g_{1} T+g_{2} T^{2}+g_{3} T^{3}
$$

at the temperature of interest, $T\left({ }^{\circ} \mathrm{C}\right)$ whereas $E_{0}$ is that at absolute zero temperature where thermal vibration energy is absent, $R$ is gas constant $(8.3145 \mathrm{~J} / \mathrm{mol} / \mathrm{K})$ and $T_{a b s}$ is absolute temperature in $\mathrm{K}$. Both terms in eq. (1) are the functions of temperature and inelastic strain rate, $\dot{\varepsilon}_{i n}$. The first term of eq.(1) represents the short-range component characterized by its thermal activation energy $Q_{s r}$ and the reference strain rate, $\dot{\varepsilon}_{s 0}=10^{8} / \mathrm{s}$ whereas the long-range component is described by the second term with its thermal activation energy $Q_{l r}$ and two additional constants, $\dot{\varepsilon}_{10}$ and $n$. The functional form of the former is the one proposed by Mecking and Kocks (1981) but that of the latter is newly introduced in this study in order to express the behaviour showing the power-law dependency, $\sigma \propto \dot{\varepsilon}_{i n}^{1 / n}$ at low stress level but asymptotically approaching to the upper bound value, $\left(E / E_{0}\right) \sigma_{l r, \max }$, as the inelastic strain rate grows. The short-term component decreases with the increase of temperature or decrease of strain rate and becomes negligible at intermediate temperatures between room temperature and creep region at typical strain rates of practical interest. On the other hand, the long-term component does not show a change until a certain combination of temperature and strain rate is reached. As a result, there appear three regions, in terms of strain rate-dependency of the yield stress, i.e. low temperature rate-dependent region due to the variation of the short-term component, high temperature rate-dependent region coming from the variation of the long-term component and the intermediate region where the yield stress is virtually rate-independent.

It is also well-known that there is a region where the inverse dependency on temperature and strain rate is observed in an intermediate temperature range and this is attributed to dynamic strain aging due to the pinning of dislocations by atoms diffused by thermal activation. An additional parameter, $R_{D S A}$, is introduced to include this effect by

$$
R_{D S A}=\lambda \exp \left[-\left(\ln \left(\dot{\varepsilon}_{i n} / \dot{\varepsilon}_{D S A}\right)+Q_{D S A} / R T_{a b s}\right)^{2} / Z\right]
$$

using its thermal activation energy, $Q_{D S A}$ and the reference strain rate, $\dot{\varepsilon}_{D S A}$ as well as $\lambda$ and $Z$ representing the maximum magnitude and the extension of stress increase due to dynamic strain aging, 
respectively.

\section{Modeling of Strain Hardening and Softening}

Most metallic materials show strain hardening characterized by increase of stress with inelastic strain except very high temperature and this modeling is indispensable for accurate evaluation of deformation behaviour at a wide range of loading condition. Strain hardening is expressed by the slope of stressinelastic strain curve, $d \sigma / d \varepsilon_{\text {in }}$ but this value is influenced by many parameters, as the initial yield stress does. It is known that strain hardening occurs as a result of change of microstructures such as increase of dislocation density or subgrain structures so that this is considered as an increase of long-range component and fairly insensitive of temperature and strain rate until the recovery effect becomes significant at higher temperatures. In this study, variation of hardening rate was approximated by the following equation combining strain hardening and strain softening:

$$
\frac{d \sigma}{d \varepsilon_{i n}}=\sigma_{0}^{m}\left[\frac{1}{m \sigma^{m-1}}-\frac{\left\{\left(1+A \varepsilon_{i n}\right) \sigma\right\}^{p}}{\sigma_{r}^{m+p-1}}\right]
$$

where $\sigma_{0}$ and $m$ are constants when the stress-inelastic relation is approximated by a pseudo-power law relation alone as

$$
\varepsilon_{i n}=\frac{\sigma^{m}-\sigma_{y}^{m}}{\sigma_{0}^{m}}
$$

with the yield stress, $\sigma_{y}$ corresponding to $\varepsilon_{i n}=0$, with the absence of the second term in the parenthesis.

The second term representing strain softening more explicitly becomes necessary at high temperatures and $\sigma_{r}$ and $p$ are parameters controlling its characteristics. The amount of hardening can be suppressed by letting its contribution increase as a function of temperature and strain rate. Steady-state stress is obtained as the stress where the first and second terms become equal in opposite signs. A coefficient in the second term, $\left(1+A \varepsilon_{i n}\right)$ was used to represent the gradual softening observed in SQV2A particularly and softening rate increases by the amount of accumulated inelastic strain, $\varepsilon_{\text {in }}$, multiplied by the constant, $A$.

Both $\sigma_{0}$ and $\sigma_{r}$ were assumed to change with temperature and/or strain rate according to

$$
\begin{gathered}
\sigma_{0}=\operatorname{Min}\left[h_{0}+h_{1} T^{\prime}+h_{2} T^{\prime 2}+h_{3} T^{\prime 3}+h_{4} T^{\prime 4}+h_{5} T^{\prime 5}, \exp \left(h_{6}+h_{7} \Omega\right)\right] \\
T^{\prime}=\min \left(T, T_{\max }\right) \\
\Omega_{h}=\ln \left(\dot{\varepsilon}_{i n}\right)+Q_{h} / R T_{a b s} \\
\sigma_{r}=\exp \left(s_{0}+s_{1} \Omega_{r}+s_{2} \Omega_{r}^{2}+s_{3} \Omega_{r}^{3}\right) \\
\Omega_{r}=\ln \left(\dot{\varepsilon}_{i n}\right)+Q_{r} / R T_{a b s}
\end{gathered}
$$

using Zener-Holloman-type parameter with the activation energies for each contribution, $Q_{h}$ and $Q_{r}$ for representing the effects of temperature and inelastic strain rate in a unified way.

\section{Determination of Constants}

Constants of the model were determined by the following procedures.

(i) Stress-inelastic strain relation in each test is approximated by eq.(4) with fixed values of $m$ and $p$.

(ii) Temperature- and strain rate-dependency of yield stress is approximated by eq. (1) with eq.(3).

(iii) Temperature- and strain rate-dependency of $\sigma_{0}$ and $\sigma_{r}$ is approximated by eqs.(6) -(10).

Solver function in EXCEL was used through all procedures to search for optimized values with the 
smallest residual.

Constants obtained following these procedures for three materials are summarized in Table 1 . It should be noted that two sets of constants were obtained for SQV2A because the characteristics of stressstrain curves changed between $700^{\circ} \mathrm{C}$ and $800^{\circ} \mathrm{C}$, probably due to phase transformation happening at a certain temperature during the course of heating.

\section{COMPARISON OF SIMULATIONS AND ACTUAL DATA}

\section{Temperature and Strain-Rate Dependency of Yield Stress}

Figure 1 shows the comparisons of test results and model predictions regarding the temperaturedependency of the yield stress at four strain rates. Model predictions are in generally good agreement with the test data for a quite wide range of temperature, although there seems to be some scattering in the test data.

Figure 2 illustrates how the yield stress at each temperature changes with a large change of strain rate in the current modelling, in comparison with the test data obtained at limited strain rates. It can be seen that the regions where strain rate-dependency appears due to short-range component and those due to long-range component are separated at all the temperature examined due to the difference in the activation energy of each component but their distances become smaller as the temperature increases..

\section{Temperature and Strain-Rate Dependency of Hardening Parameters}

Then Figure 3 and Figure 4 show the temperature-dependency of the hardening and softening parameters at four strain rates, respectively. Both parameters decrease with the increase of temperature and/or decrease of strain rate in all materials, except SQV2A between $700^{\circ} \mathrm{C}$ and $800^{\circ} \mathrm{C}$ where the deformation behavior changes discontinuously due to phase transformation.

\section{Comparison of Stress-Strain Curves}

Finally whole stress-inelastic strain curves predicted by the model are compared with the test data. Figure 5 examines the temperature-dependency of the curves fixing the strain rate at $0.001 \mathrm{~s}^{-1}$ whereas the strain rate-dependency is examined at the temperature of $700^{\circ} \mathrm{C}$ in Figure 6, where the strain rate-effect is quite large in all materials examined. Although not perfect, test data and model predictions seem to be in reasonable agreement in both figures, suggesting the applicability of the common framework of the constitutive model as well as the effectiveness of the way to identify the constants for each alloy having fairly different characteristics.

\section{CONCLUDING REMARKS}

In consideration of the application to structural analysis under BDBE condition, modelling of inelastic deformation behaviour at a wide range of temperatures and strain rate has been trialled in this study. Constitutive models fundamentally based on underlying physical mechanism leading to temperature- and strain rate-dependency have been formulated and applied to three alloys used in light water reactor plants. It has been demonstrated that temperature- and strain rate-dependency can be described in a unified manner by the proposed equations with a reasonable accuracy. Extension to nonisothermal conditions needs to be studied as a next step towards the establishment of models applicable to structural analysis. Development of more general formulation including the division of the hardening into isotropic and kinematic elements would be another challenge for improving the capability.

\section{REFERENCES}


Mecking, H. and Kocks, U. F. (1981) "Kinetics of flow and strain hardening," Acta Metallurgica, 29, 1865-1875.

Table 1 Summary of Constants in Constitutive Models

\begin{tabular}{|c|c|c|c|c|c|}
\hline \multirow{2}{*}{\multicolumn{2}{|c|}{$\frac{\text { Material }}{\text { Temperature }}$}} & \multicolumn{2}{|c|}{ SQV2A } & \multirow{2}{*}{$\begin{array}{l}\text { SUS316 } \\
\text { All range }\end{array}$} & \multirow{2}{*}{$\begin{array}{l}\text { NCF600 } \\
\text { All range }\end{array}$} \\
\hline & & $T<800^{\circ} \mathrm{C}$ & $T \geqq 800^{\circ} \mathrm{C}$ & & \\
\hline \multirow{5}{*}{$\begin{array}{l}\text { Young's } \\
\text { modulus }\end{array}$} & $g_{0}$ & \multicolumn{2}{|c|}{205,000} & 197,700 & 214,200 \\
\hline & $g_{1}$ & \multicolumn{2}{|c|}{64.29} & -59.19 & -38.65 \\
\hline & $g_{2}$ & \multicolumn{2}{|c|}{$-3.248 \times 10^{-1}$} & $2.665 \times 10^{-2}$ & $-3.164 \times 10^{-2}$ \\
\hline & $g_{3}$ & \multicolumn{2}{|c|}{$1.449 \times 10^{-4}$} & 0 & 0 \\
\hline & $E_{0}(\mathrm{MPa})$ & \multicolumn{2}{|c|}{230,000} & 211,700 & 221,900 \\
\hline \multirow{2}{*}{$\begin{array}{l}\text { Short-range } \\
\text { yield stress }\end{array}$} & $\sigma_{s r, \text { max }}(\mathrm{MPa})$ & \multicolumn{2}{|c|}{1344} & 472.2 & 420.8 \\
\hline & $Q_{s r}(\mathrm{~J} / \mathrm{mol})$ & \multicolumn{2}{|c|}{73,160} & 174,500 & 429,700 \\
\hline \multirow{4}{*}{$\begin{array}{l}\text { Long-range } \\
\text { yield stress }\end{array}$} & $\sigma_{I r, \text { max }}(\mathrm{MPa})$ & \multicolumn{2}{|c|}{506.4} & 151.9 & 429.7 \\
\hline & $\dot{\varepsilon}_{10}\left(\mathrm{~S}^{-1}\right)$ & $1.683 \times 10^{12}$ & $1.00 \times 10^{9}$ & $4.407 \times 10^{14}$ & $2.938 \times 10^{22}$ \\
\hline & $n$ & 2.687 & 4.440 & 4.459 & 5.303 \\
\hline & $Q_{l r}(\mathrm{~J} / \mathrm{mol})$ & 265,700 & 196,500 & 434,300 & 518,300 \\
\hline \multirow{4}{*}{$\begin{array}{l}\text { Dynamic } \\
\text { strain } \\
\text { aging }\end{array}$} & $\dot{\varepsilon}_{D S A}\left(\mathrm{~s}^{-1}\right)$ & \multicolumn{2}{|l|}{ - } & $3.871 \times 10^{74}$ & $4.730 \times 10^{49}$ \\
\hline & $Z$ & \multicolumn{2}{|l|}{ - } & 145.5 & 321.8 \\
\hline & $\lambda$ & \multicolumn{2}{|l|}{-} & 0.5500 & 1.440 \\
\hline & $Q_{D S A}(\mathrm{~J} / \mathrm{mol})$ & \multicolumn{2}{|l|}{-} & $1,538,000$ & $1,281,000$ \\
\hline \multirow{11}{*}{$\begin{array}{c}\text { Strain } \\
\text { hardening }\end{array}$} & $m$ & 5.357 & 2.000 & 2.000 & 2.721 \\
\hline & $h_{0}$ & \multicolumn{2}{|c|}{1,063} & 1,279 & 1,513 \\
\hline & $h_{1}$ & \multicolumn{2}{|c|}{$-6.703 \times 10^{-1}$} & $3.476 \times 10^{-1}$ & $2.461 \times 10^{-1}$ \\
\hline & $h_{2}$ & \multicolumn{2}{|c|}{$5.470 \times 10^{-3}$} & $-1.575 \times 10^{-2}$ & $-1.904 \times 10^{-3}$ \\
\hline & $h_{3}$ & \multicolumn{2}{|c|}{$-1.271 \times 10^{-5}$} & $7.469 \times 10^{-5}$ & $1.829 \times 10^{-6}$ \\
\hline & $h_{4}$ & \multicolumn{2}{|c|}{$8.632 \times 10^{-9}$} & $-1.333 \times 10^{-7}$ & $-6.599 \times 10^{-10}$ \\
\hline & $h_{5}$ & \multicolumn{2}{|c|}{0} & $8.020 \times 10^{-11}$ & 0 \\
\hline & $T_{\max }\left({ }^{\circ} \mathrm{C}\right)$ & \multicolumn{2}{|c|}{500} & 600 & 1100 \\
\hline & $Q_{h}(\mathrm{~J} / \mathrm{mol})$ & 399,700 & 305,800 & 380,500 & - \\
\hline & $h_{6}$ & -3.049 & 2.204 & 0.1028 & - \\
\hline & $h_{7}$ & 0.2108 & 0.1313 & 0.1745 & - \\
\hline \multirow[t]{7}{*}{ Softening } & $p$ & 1.326 & 3.000 & 3.000 & 3.170 \\
\hline & $s_{0}$ & -24.55 & -1.494 & -12.12 & 1.425 \\
\hline & $s_{1}$ & 1.389 & 0.2993 & 0.9050 & 0.1367 \\
\hline & $s_{2}$ & $-2.106 \times 10^{-2}$ & $-9.461 \times 10^{-4}$ & $-1.519 \times 10^{-2}$ & $-2.347 \times 10^{-4}$ \\
\hline & $s_{3}$ & $1.088 \times 10^{-4}$ & $-3.080 \times 10^{-5}$ & $8.909 \times 10^{-5}$ & 0 \\
\hline & $Q_{s}(\mathrm{~J} / \mathrm{mol})$ & 424,500 & 281,500 & 433,300 & 358,000 \\
\hline & $A$ & 3.817 & 0 & 0 & 0 \\
\hline
\end{tabular}

Note: All the values are valid when stress and inelastic strain rate are expressed in MPa and s ${ }^{-1}$. 
$24^{\text {th }}$ Conference on Structural Mechanics in Reactor Technology

BEXCO, Busan, Korea - August 20-25, 2017

Division I
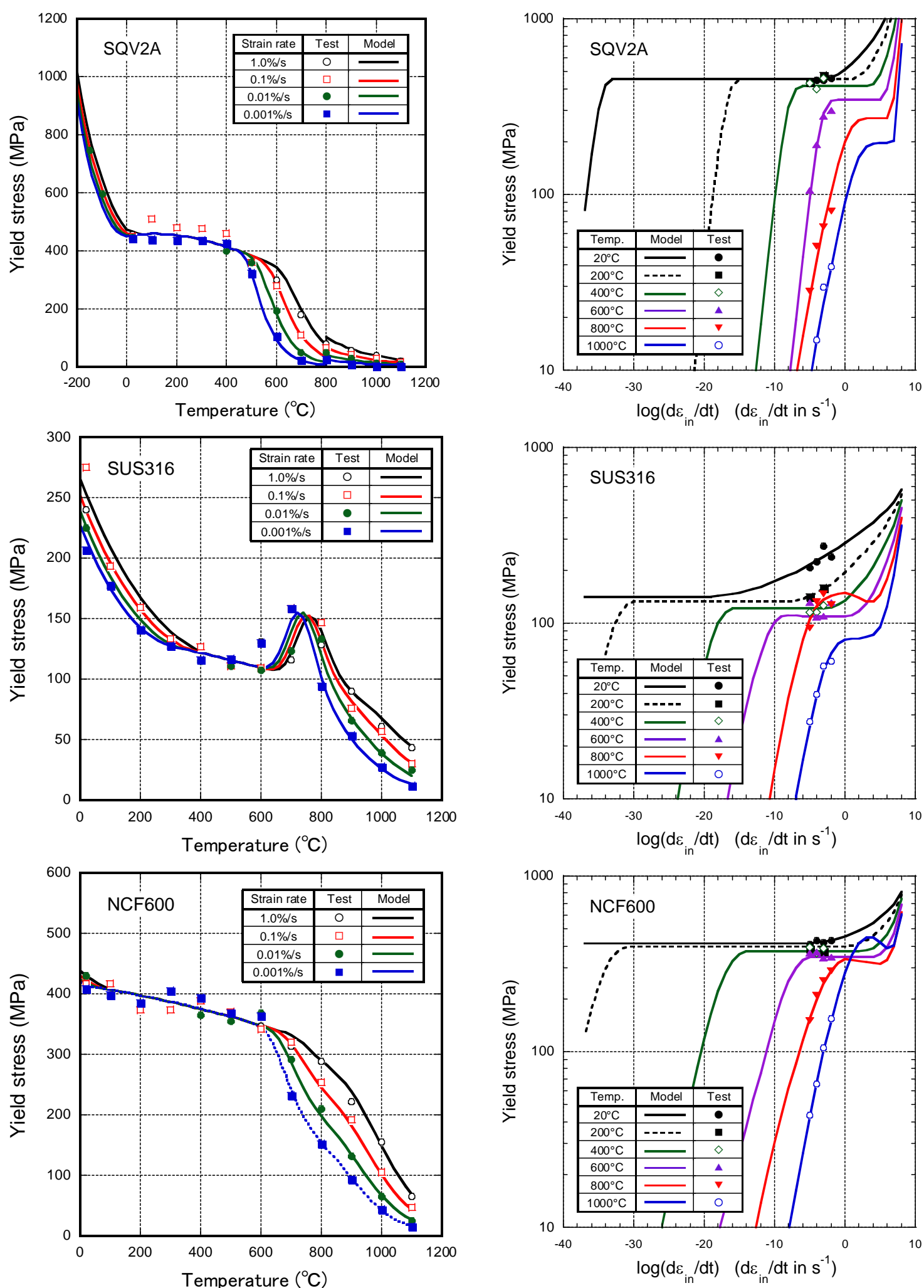

Figure 1 Comparison on temperaturedependency of yield stress

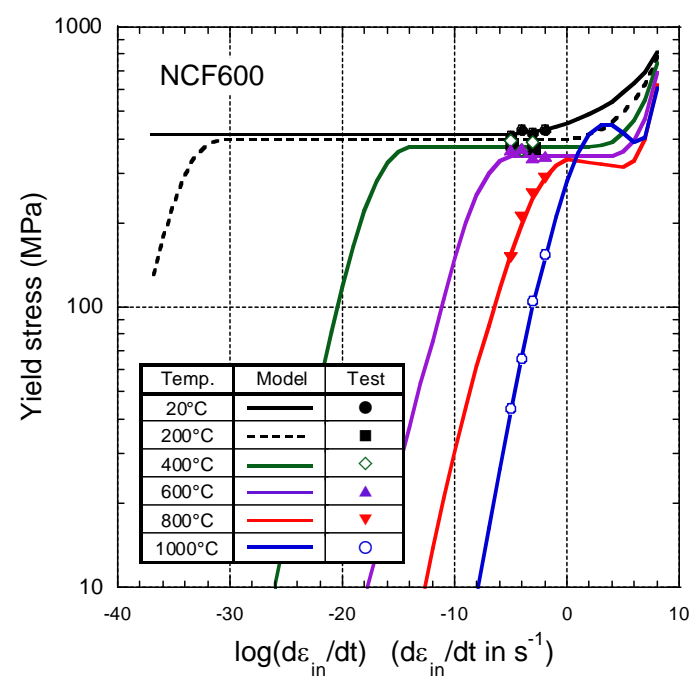

Figure 2 Comparison on strain rate-dependency of yield stress 
$24^{\text {th }}$ Conference on Structural Mechanics in Reactor Technology BEXCO, Busan, Korea - August 20-25, 2017

Division I
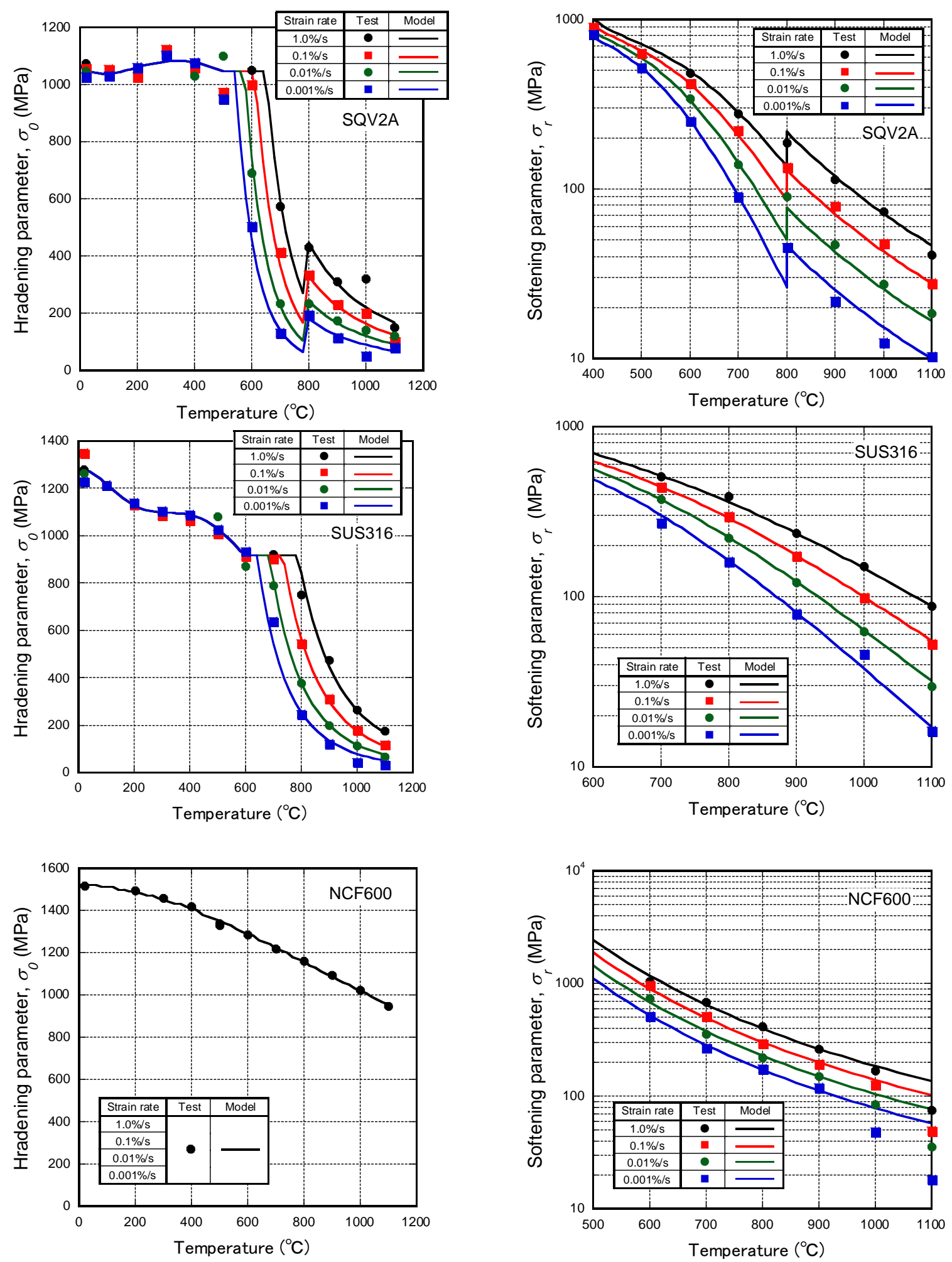

Figure 3 Temperature-dependency of hardening parameter

Figure 4 Temperature-dependency of softening parameter 

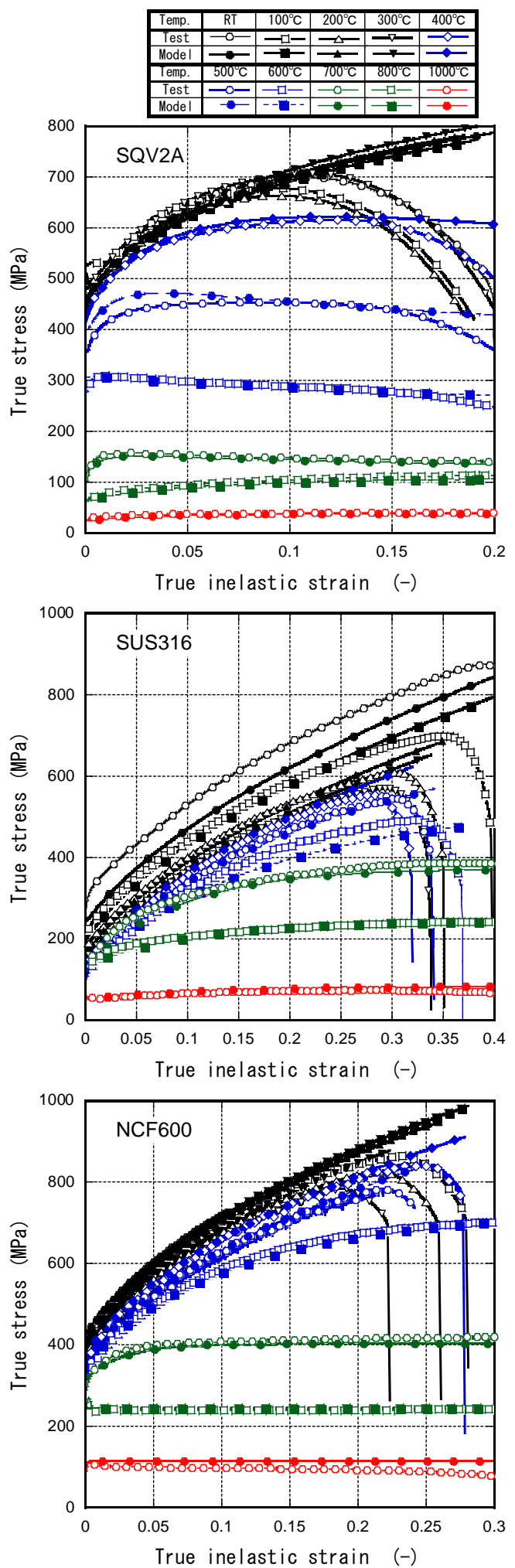

Figure 5 Comparison of experimental and predicted stress-inelastic strain curves at various temperatures (strain rate:0.001/s)
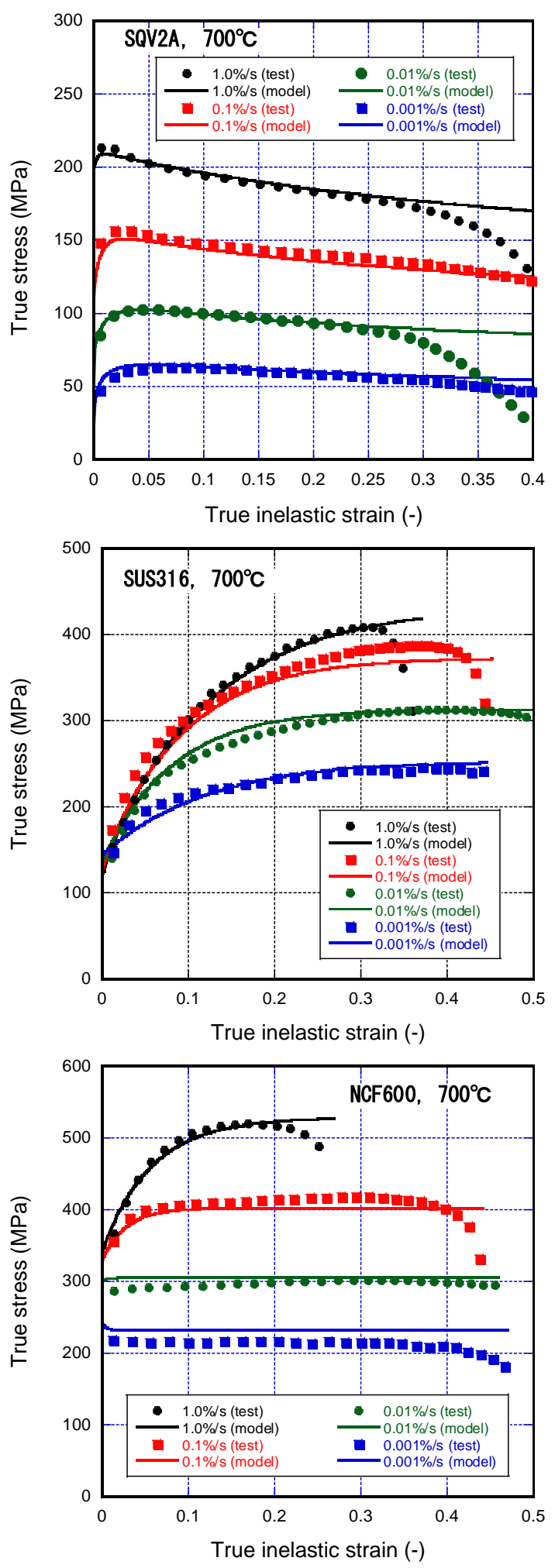

Figure 6 Comparison of experimental and predicted stress-inelastic strain curves at various strain rates $\left(700^{\circ} \mathrm{C}\right)$ 